\title{
Analysis of Motion Self-Occlusion Problem Due to Motion Overwriting for Human Activity Recognition
}

\author{
Md. Atiqur Rahman Ahad, Joo Kooi Tan, Hyoung Seop Kim and Seiji Ishikawa \\ Faculty of Engineering, Kyushu Institute of Technology, Fukuoka, Japan \\ Email: atiqahad@ss10.cntl.kyutech.ac.jp
}

\begin{abstract}
Various recognition methodologies address to recognize and understand varieties of human activities. However, motion self-occlusion due to motion overlapping in the same region is a daunting task to solve. Various motion-recognition methods either bypass this problem or solve this problem in complex manner. Appearance-based template matching paradigms are simpler and hence these approaches faster for activity analysis. In this paper, we concentrate on motion selfocclusion problem due to motion overlapping in various complex activities for recognition. In the Motion History Image (MHI) method, the self-occlusion is evident and it should be solved. Therefore, this paper compares our directional motion history image concept with basic the Motion History Image, Multi-level Motion History representation and Hierarchical Motion History Histogram representation to solve the self-occlusion problem of basic the Motion History Image representation. We employ some complex aerobics and find the robustness of our method compared to other methods for this self-occlusion problem. We employ seven higher order $\mathrm{Hu}$ moments to compute the feature vector for each activity. Afterwards, $k$-nearest neighbor method is utilized for classification with leave-one-out paradigm. The comparative results clearly demonstrate the superiority of our method than other recent approaches. We also present several experiments to demonstrate the performance and strength of the DMHI method in recognizing various complex actions.
\end{abstract}

Index Terms-MHI, DMHI, MMHI, HMHН, motion recognition, feature vector

\section{INTRODUCTION}

Human motion and behavior analysis is very important area in computer vision. There are many approaches to deal with action or activity recognition and analysis. We concentrated on the MHI method [1] as it is an appearance-based templatematching motion recognition approach. It has been widely employed and modified a bit based on the requirements [2]. Various interactive systems have been successfully constructed using motion history template as a primary sensing mechanism.

Refs. [2-5] cover some dominant methods for activity analysis and recognition. We see that most of the approaches consider simple or one-directional actions (e.g., sitting on a chair, standing from a chair, raise a hand, etc.) for recognition. However, usual activities are complex in nature and not simple in dimension. By complex action or activity, it is meant that the action comprises more than one action primitives or basic action elements. Hence, a 'sit-stand' action is a complex action, because it has 'sitting motion' and 'standing motion' in two directions (down and up direction consecutively). Reallife activities are complex in nature. In this paper, we discuss the motion recognition problem of complex action that has motion overwriting problem. Motion overwriting can be defined using the 'sit-stand' action, because a 'standing' action after a 'sitting-down' action will erase or passes the prior motion information of the 'sitting-down' action. In this paper, we start with the Motion History Image (MHI) method as it is found to be robust in recognition and simple in computation. However, this method can not solve the overwriting or motion self-occlusion problem $[6,8]$.

The paper is organized as follows: Section II presents some related works on activity recognition. Section III covers the basic MHI method and the concept of overwriting problem. Then we present three template-based representations including our method to solve the self-occlusion problem in Section IV. The recognition methods based on these motion representations are outlined in Section V. Section VI presents the comparative results and analysis using recognition rates and confusion matrices for various aerobics sequences. This section also illustrates the robustness of the DMHI method, performances on low-resolution video sequences and the concept of reduced dimensional feature vectors. Finally, Section VII concludes the paper with some future guidelines.

\section{RELATED WORKS AND MOtIVATION}

This paper presents four methods along with the MHI method, which is very widely employed method for various applications. For example, using the MHI method, Davis et al. [9] have developed a virtual aerobics trainer that watches and responds to a user as he/she performs a workout. An interactive art demonstration can be constructed from the motion templates [10]. An interactive and narrative play space for children, called KidsRoom was developed using the MHI method successfully. Yau et al. [11] have developed a method for visual speech recognition employing the MHI method. The video data of the speaker's mouth is represented using grayscale images named as motion history image. Automatically localizing and tracking moving person or vehicle for an automatic visual surveillance system was demonstrated in [12-13] by employing the MHI method before employing an extended mean shift approach in [13]. Son et al. 
[14] have calculated the MHI and then combined with background model to detect candidate road image.

Gait History Image and Gait Energy Image are created for gait analysis based on the concept of the MHI [15-16]. Ref. [17] has proposed a threat assessment method for automated visual surveillance with the aid of the MHI. A PDA-based recognition system based on the MHI method is developed by [18]. Petras et al. [19] have devised a flexible test-bed for unusual behavior detection and automatic event analysis using the MHI. A recent method by Kellokumpu et al. [20] have proposed a recognition method at the top of the MHI method using texture-based description of the movements by employing local binary pattern (LBP) operator. Later they have used HMM for recognition. Usually, the MHI-based methods consider global image in the scene, rather body parts. However, they have split the MHI image into four regional parts to compute histograms of those areas.

Ahad et al. [2] have surveyed various action and activity recognition approaches since 2001 till 2008. This paper sums up different key methods along with the challenges ahead for researchers to solve in activity analysis and recognition. One of the important challenges is to solve the motion selfocclusion problem due to motion overwriting in activity recognition. The basic MHI method has failed to address this problem and therefore, new concepts are obvious to mitigate this fundamental problem of motion recognition. The following sections address this issue.

\section{Motion History IMAge Template}

The MHI is a scalar-valued image where more recently moving pixels are brighter (i.e., the presence of high intensity pixels), and vice versa (i.e., a low intensity pixel denotes a motion that occurred earlier in time). And intensity zero denotes no motion at all at that specific location. The MHI [1] expresses the motion flow or sequence by using the intensity of every pixel in temporal manner. To describe the motionshape and spatial distribution of motion, the MHI method has also introduced the Motion Energy Image (MEI) or binary motion region. One of the advantages of the $\mathrm{MHI}$ representations is that a range of times from frame to frame to several seconds may be encoded in a single frame, and in this way, the MHI spans the time scale of human gesture. The MHI $H_{\tau}(x, y, t)$ can be computed from an update function $\Psi(x, y, t)$ :

$$
H_{\tau}(x, y, t)=\left\{\begin{array}{cc}
\tau & \text { if } \Psi(x, y, t)=1 \\
\max \left(0, H_{\tau}(x, y, t-1)-\delta\right) & \text { otherwise }
\end{array}\right.
$$

Here, $(x, y)$ and $t$ show the position and the time respectively; $\Psi(x, y, t)$ signals object presence (or motion) in the current video image; $\tau$ decides the temporal duration of the MHI (e.g., in terms of frames); and $\delta$ is the decay parameter. This update function is called for every new video frame analyzed in the sequence. Some possible image processing techniques for defining this update function are background subtraction, image differencing, optical flow, etc. The MHI is generated from an image, obtained from frame subtraction, using a threshold $\xi$ :

$$
\Psi(x, y, t)=\left\{\begin{array}{lc}
1 & \text { if } D(x, y, t) \geq \xi \\
0 & \text { otherwise }
\end{array}\right.
$$

where, $D(x, y, t)$ with difference distance $\Delta$ is:

$$
D(x, y, t)=|I(x, y, t)-I(x, y, t \pm \Delta)|
$$

Here, $I(x, y, t)$ is the intensity value of pixel location with coordinate $(x, y)$ at the $t^{\text {th }}$ frame of the image sequence. Two neighboring frames can be described by [13]:

$$
\begin{aligned}
& I(x, y, t)=b_{t}(x, y)+m_{t}(x, y)+\varepsilon_{t}(x, y) \\
& I(x, y, t+1)=b_{t+1}(x, y)+m_{t+1}(x, y)+\varepsilon_{t+1}(x, y)
\end{aligned}
$$

where, $b_{t}(x, y)$ is the static background for the $t^{t h}$ frame, $m_{t}(x, y)$ is moving objects for the $t^{\text {th }}$ frame and $\varepsilon_{t}(x, y)$ is the background noise for the $t^{\text {th }}$ frame. Therefore, $D(x, y, t)$ can be redefined as,

$$
D(x, y, t)=b(x, y)+m_{t}^{\partial}(x, y)+\varepsilon_{t}^{\partial}(x, y)
$$

where, $b(x, y)$ is the overlapped area in consecutive frames, $m_{t}^{\partial}(x, y)$ is the motion area and $\varepsilon_{t}^{\partial}(x, y)$ is the noise. Obviously, $D(x, y, t)$ contains part of the moving objects, background aberration due to motion and noise which lead to incorrect results, such as over-segment, motion ambiguity, and distortion. Moreover, motion at low speed can not be easily detected [13]. Now, we will compute the MEI. As stated above, the $\mathrm{MHI}$ is a scalar-valued image where more recently moving pixels are brighter, and vice versa; whereas, the MEI is a binary image, generated by thresholding the MHI above zero:

$$
E_{\tau}(x, y, t)=\left\{\begin{array}{lc}
1 & \text { if } H_{\tau}(x, y, t) \geq 1 \\
0 & \text { otherwise }
\end{array}\right.
$$
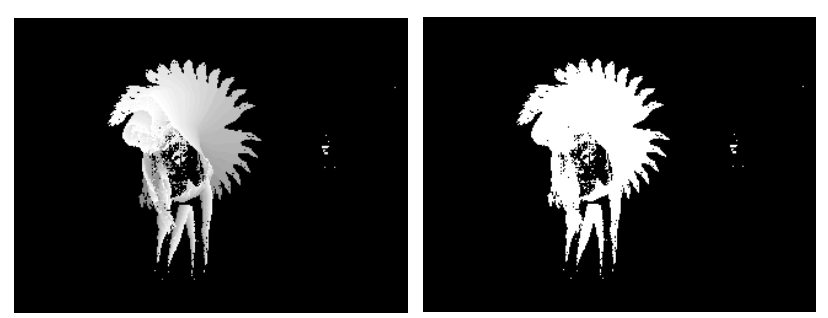

Figure 1. (a) The MHI (left image); (b) The MEI (right) for an action.

Fig. 1 shows the MHI and the MEI for a hand-waving action. However, the MHI can not solve motion self-occlusion or overwrite problem, since previous motion is deleted or overwritten by the later motion information, if the motion has opposite directions in action (e.g., if a person sits down, and then stands up, the final MHI image should contain brighter pixels in the upper part of the image to represent the stand-up motion). This self-occlusion of the moving object or person overwrites the prior information [6]. Hence, for complex actions or activities, the basic MHI method can not recognize properly due to its inherent nature of motion history calculation. We developed a robust method considering four compass directions (namely, up, down, left and right 
directions) of an action, and thereby we can simply separate an action into its directional elements. This method is called Directional Motion History Image (DMHI) method [34]. This method is based on the computation of optical flow. The DMHI method outperforms the basic MHI method. We extend the evaluation with other representation methods that claim to be a solution for such motion overwriting problem. We evaluate recent methods, called the Hierarchical Motion History Histogram $(\mathrm{HMHH})$ representation $[8,21,22]$ and the Multi-level Motion History Image (MMHI) method [24], along with the MHI and the DMHI methods.

\section{TEMPlate-BASED REPRESENTATIONS TO SOLVE MOTION SELF-OCCLUSION PROBLEM}

\section{A. Directional Motion History Image Representation}

The Directional Motion History Image (DMHI) method can solve the motion-overwriting problem due to self-occlusion (which is inherent in the MHI method) in human activities. We will see that it can solve the overwriting problem smartly. Fig. 2 demonstrated the motion overwrite problem using the MHI and the DMHI approaches. Using the DMHI method, the problem can be solved significantly.

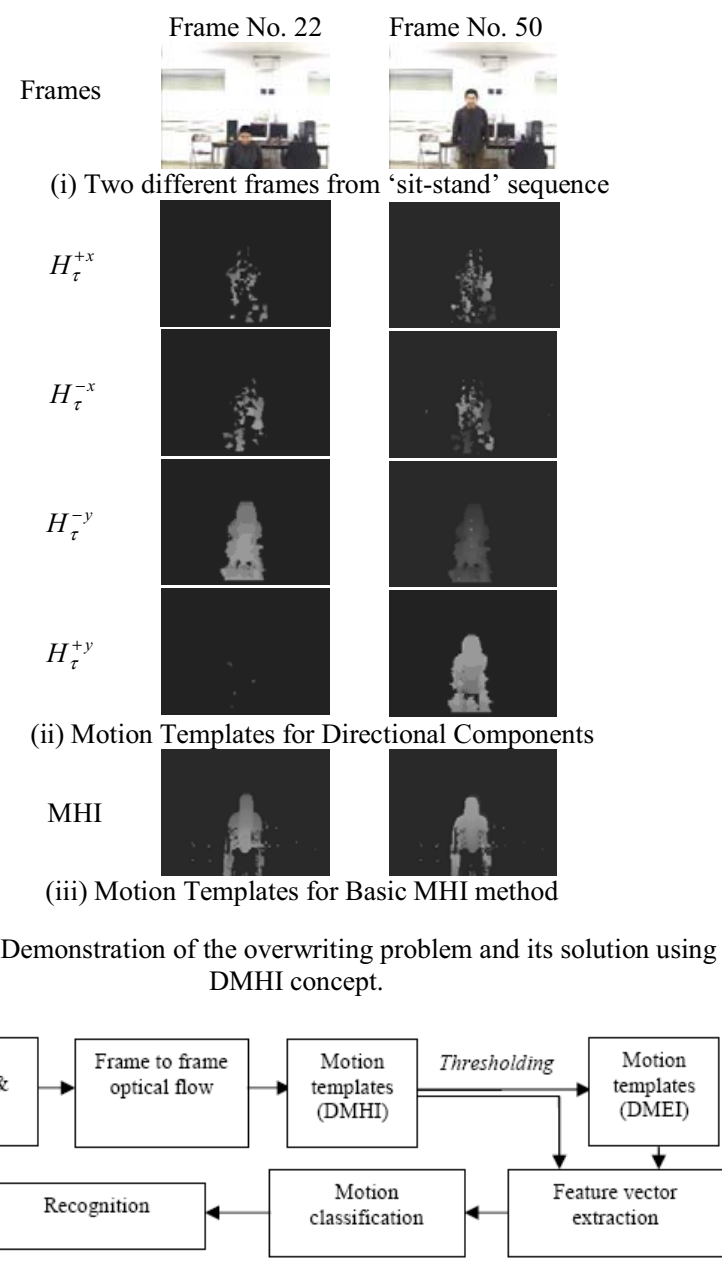

Figure 3. System flow diagram for the DMHI method.
Fig. 3 illustrates the system flow-diagram of this method up to the recognition of activities. In this approach, instead of background or frame subtraction, gradient-based optical flow vector [32], $\Psi(x, y, t)$ is calculated between consecutive two frames and split it into four channels (as depicted in Fig. 4), based on the concept of motion descriptors, based on smoothed and aggregated optical flow measurements in four channels [27]. These four non-negative channels are,

$$
\begin{aligned}
& \Psi_{x}(x, y, t)=\Psi_{x}^{+}(x, y, t)-\Psi_{x}^{-}(x, y, t) \\
& \Psi_{y}(x, y, t)=\Psi_{y}^{+}(x, y, t)-\Psi_{y}^{-}(x, y, t)
\end{aligned}
$$

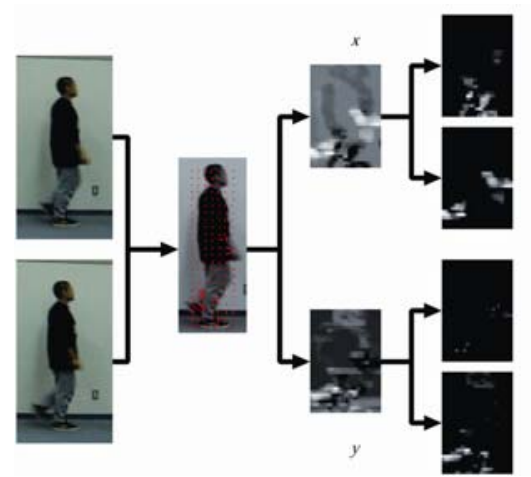

Figure 4. Optical flow is split into four different channels.

In this way, we get four-directional motion templates for left, right, up and down directions based on a threshold $\zeta$. We calculate the corresponding four history images as follows,

$$
\begin{aligned}
H_{\tau}^{-x}(x, y, t) & =\left\{\begin{array}{cc}
\tau & \text { if } \Psi_{x}^{-}(x, y, t)>\zeta \\
\max \left(0, H_{\tau}^{-x}(x, y, t-1)-\delta\right) & \text { otherwise }
\end{array}\right. \\
H_{\tau}^{+x}(x, y, t) & =\left\{\begin{array}{cc}
\tau & \text { if } \Psi_{x}^{+}(x, y, t)>\zeta \\
\max \left(0, H_{\tau}^{+x}(x, y, t-1)-\delta\right) & \text { otherwise }
\end{array}\right. \\
H_{\tau}^{-y}(x, y, t) & =\left\{\begin{array}{cc}
\tau & \text { if } \Psi_{y}^{-}(x, y, t)>\zeta \\
\max \left(0, H_{\tau}^{-y}(x, y, t-1)-\delta\right) & \text { otherwise }
\end{array}\right. \\
H_{\tau}^{+y}(x, y, t) & =\left\{\begin{array}{cc}
\tau & \text { if } \Psi_{y}^{+}(x, y, t)>\zeta \\
\max \left(0, H_{\tau}^{+y}(x, y, t-1)-\delta\right) & \text { otherwise }
\end{array}\right.
\end{aligned}
$$

Moreover, the corresponding energy images are calculated as,

$$
\begin{aligned}
& E_{\tau}^{-x}(x, y, t)=\left\{\begin{array}{lc}
1 & \text { if } H_{\tau}^{-x}(x, y, t)>0 \\
0 & \text { otherwise }
\end{array}\right. \\
& E_{\tau}^{+x}(x, y, t)=\left\{\begin{array}{lc}
1 & \text { if } H_{\tau}^{+x}(x, y, t)>0 \\
0 & \text { otherwise }
\end{array}\right. \\
& E_{\tau}^{-y}(x, y, t)=\left\{\begin{array}{lc}
1 & \text { if } H_{\tau}^{-y}(x, y, t)>0 \\
0 & \text { otherwise }
\end{array}\right. \\
& E_{\tau}^{+y}(x, y, t)=\left\{\begin{array}{lc}
1 & \text { if } H_{\tau}^{+y}(x, y, t)>0 \\
0 & \text { otherwise }
\end{array}\right.
\end{aligned}
$$

This DMHI approach can solve the motion overwriting problem significantly, and hence performs well for repetitive or complex activities. These eight motion templates are required for further motion representations. Using only history images or energy images for motion representation are not worthy. It has been proved both theoretically and experimentally that both templates are required for better motion representations [1]. Though some researchers employ the history images only for their research, they employ or implement some other features along with the history image to 
represent the motion sequence in a robust way [35]. However, to use only energy image for representation makes no real sense due to the fact that energy image gives the total motion region in the full video sequence. To make it clear, let's consider the 'stand-up' and 'sit-down' motion sequence. In both reverse directions, we will get almost similar energy images (i.e., for 'sit' and then 'stand' sequence; or 'stand' and then 'sit' sequence), and hence energy image only can not give good cue unless the motions or actions are very much different among each other. E.g., actions like walk, jump on a place, raise hands, sleep on the floor, etc., have different dimensions and will give different energy images to separate these actions. Therefore, to accommodate various activities, similar to the MHI method, the motion energy and the motion history templates are utilized $[1,34,35]$.

\section{B. Multi-level Motion History Image Representation}

The Multiple-level MHI (MMHI) aims at overcoming the problem of motion self-occlusion by recording motion history at multiple time intervals (i.e., multi-level MHIs) [24,23]. They 'believe that MMHI data representation can offer many benefits in applications where confusions caused by motion self-occlusion are common (e.g., in hand gesture recognition, waving the hand is often confused with moving the hand from left to right only)' [24]. Initially, the input image sequences may have different numbers of frames. So, while the MHIs are temporally normalized, the number of history levels in them may still differ from one image sequence to other. To be able to compare the sequences properly, the MMHI method tries to create all MHIs such that they have a fixed number of history levels $n$. Therefore, each image sequence is sampled to $(n+1)$ frames. The modified MHI is:

$$
\operatorname{MMHI}_{t}(x, y, t)=\left\{\begin{array}{cc}
s^{*} t & \text { if } \Psi(x, y, t)=1 \\
\operatorname{MMHI}_{t}(x, y, t-1) & \text { otherwise }
\end{array}\right.
$$

where $s=(255 / n)$ is the intensity step between two history levels and $\operatorname{MMHI}_{t}(x, y, t)=0$ for $t \leq 0$. The final image is found by iteratively computing Eq. (10) for $t=1, \ldots, n+1$. The number of history levels was experimentally determined to be the number that, when used to construct the motion history templates, results in the highest recognition rate. The MMHI approach focused on automatic detection of facial action units that compose expressions [24]. It requires sophisticated registration system, because all utilized image sequences must have the faces at the same position and on the same scale. They assume that the beginning and the end of a facial expression are known and that they coincide with the duration of an image sequence, hence the MMHI does not require varying $\tau$. Instead they normalize the temporal behavior by distributing the gray values in the MHI over the available range $(0-255$, assuming that we are using 8 bit gray level images). This method encodes motion occurring at different time instances on the same pixel location such that it is uniquely decodable afterwards. For this purpose, it uses a simple bit-wise coding scheme and if motion occurs at time $t$ at pixel location $(x, y)$, it adds $2^{t-1}$ to the old motion value of the MMHI as follows:

$$
\operatorname{MMHI}(x, y, t)=\operatorname{MMHI}(x, y, t-1)+\Psi(x, y, t) \cdot 2^{(t-1)}
$$

Due to this bitwise coding scheme, it is possible to separate multiple actions occurring at the same position [24]. In their approach for facial action unit detection, they have not employed the concept of MEI or other related templates. However, in your action recognition study, we have introduced the Multi-level Motion Energy Image (MMEI) template for more information to aid the recognition process to have higher recognition results. In our experiment, we generate the MMEI by thresholding the MMHI above zero according to the following equation:

$$
\text { MMEI }(x, y, t)=\left\{\begin{array}{cc}
1 & \text { if } M M H I(x, y, t) \geq 1 \\
0 & \text { otherwise }
\end{array}\right.
$$

The result does not clearly demonstrate the superiority of MMHI with respect to basic MHI in their experiment on facial action unit (AU) detection. It is reported that self-occlusion due to motion overwriting problem might be solved using this MMHI. We implemented this method for this proof.

\section{Hierarchical Motion History Histogram Representation}

A motion representation and recognition system called Hierarchical Motion History Histogram (HMHH) is proposed to solve overwrite problem of the MHI method [8]. In [21,22], few related other methods are compared $[1,25,26]$ with this HMHH representations for some actions. To realize the HMHH method, it is required to scrutinize the overwriting problem in different manner so that the HMHH method can be established coherently. Let's select the pixels $(x, y)$ on the vertical red line of Fig. 5(a); and consider that the vertical red line has the pixels from $(80,90)$ to $(140,90)$. If some action happened at frame $\kappa$ on pixel $(x, y)$, then $D(x, y, \kappa)=1$, otherwise $D(x, y, \kappa)=0$. Hence, the locations of these pixels are $(80,90),(81,90), \ldots,(140,90)$. For a pixel $(x, y)$, the motion mask $D(x, y,:)$ of this pixel is the binary sequence (here, $N+1$ is the total no. of frames) of:

$$
D(x, y,:)=\left(b_{1}, b_{2}, \ldots, b_{N}\right), \quad b_{i} \in\{0,1\}
$$

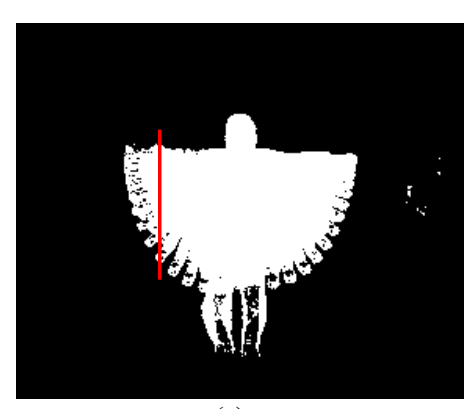

(a)

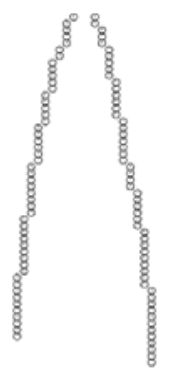

(b)
Figure 5. (a) Example of an MEI of an action sequence having hand-waving to up and then back down from up; (b) $D(:,:,:)$ on the red line is shown. The horizontal direction is the 'frame numbers' and vertical direction is the 'pixel position'. The top two circles denote the presence of motion at Frame no. 30 and then at Frame no. 33 (and in between, there is no motion occurances on that pixel location). So the later information is preserved and earlier motion information (i.e., at Frame no. 30) is abandoned in this process. 


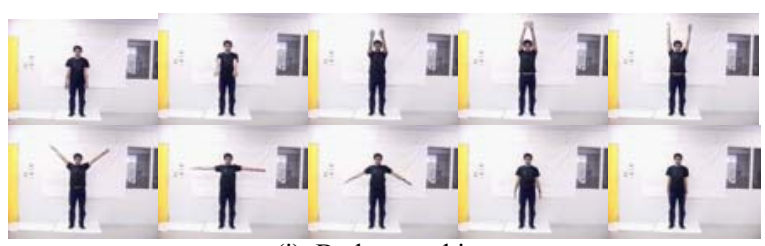

(i) Body stretching

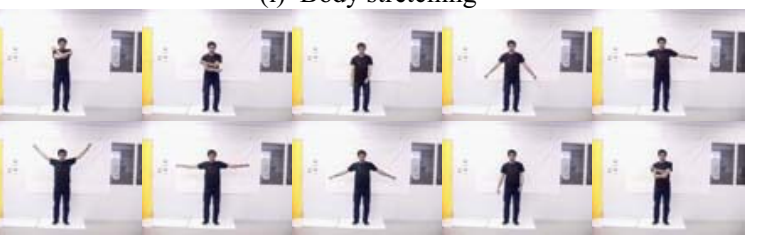

(ii) Waving arms, bending \& straighten legs

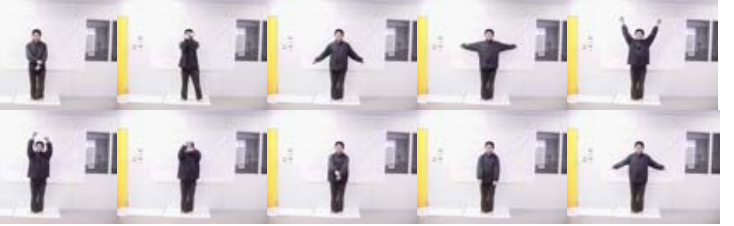

(iii) Turning the arms

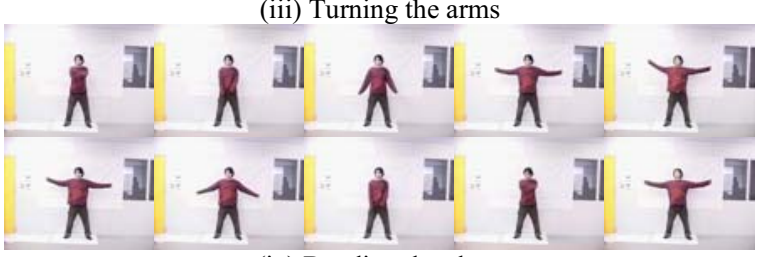

(iv) Bending the chest

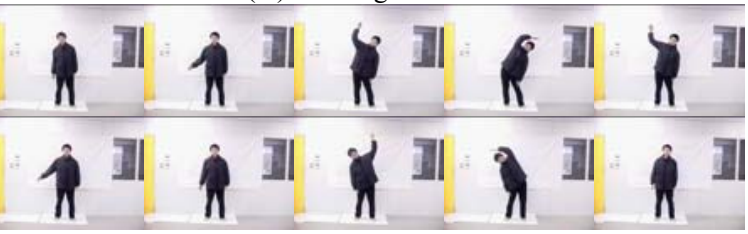

(v) Bending the body sideways

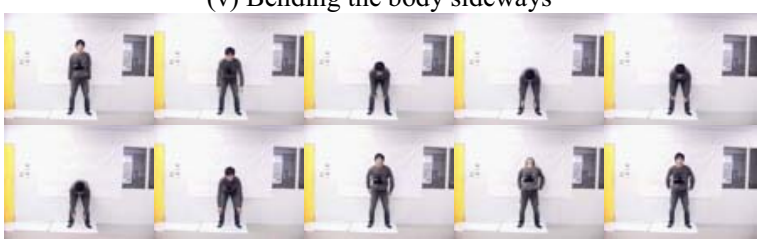

(vi) Bending the body - front and back

Figure 6. Six activities in sequential order. Activities are complex.

All of the motion masks on the red line are shown in Fig. 5 (b). Each row is $D(x, y,:)$ for one fixed pixel $(x, y)$. A white circle represents ' 1 ' or the presence of motion for that frame. Due to the formation of the MHI method, it keeps the later information of the presence of 1 (i.e., motion) in a specific pixel when there are some earlier movement in the same pixel. For example, for a pixel, the last ' 1 ' in the sequence are retained in the MHI at that pixel. It is clear that previous ' 1 ' in the sequence, when some action occurred, are not represented. And usually almost every pixel has more than one ' 1 ' in their sequence. It can be mentioned as overwriting problem in different form. To solve this problem, we can define some patterns $P_{i}$ in $D(x, y,:)$ sequences based on the number of connected ' 1 ', e.g.,

$$
P_{1}=010, P_{2}=0110, P_{3}=01110, \cdots, P_{M}=\underbrace{01 \cdots 10}_{M 1 \mathrm{~s}}
$$

Now define a subsequence $C_{i}=b_{n 1}, b_{n 2}, \ldots, b_{n i}$ and denote the set of all sub-sequences of $D(x, y,:)$ as $\Omega\{D(x, y,:)\}$. Then for each pixel $(x, y)$, lets count the number of occurrences of each specific pattern $P_{i}$ in the sequence of $D(x, y,:)$ as shown,

$$
\left.H M H H\left(x, y, P_{i}\right)=\sum_{j} \mathbf{1}_{\left\{C_{j}=P_{i} \mid C_{j} \in \Omega\{D(x, y, j)\}\right.}\right\}
$$

Here, 1 is the indicator function. Hence, from each pattern $P_{i}$, we construct one gray-scale image (called Motion History Histogram (MHH)), and in aggregation, we call all $\mathrm{MHH}$ images as Hierarchical $\mathrm{MHH}(\mathrm{HMHH}))$. In this paper, we consider four (i.e., $M=4$ ) such patterns for recognition. Hence, we get four different images from which we get feature vectors. Similar to the concept of the MEI, we can threshold each $\mathrm{MHH}$ to compute the corresponding binary energy image, called MEH:

$$
\operatorname{MEH}_{\tau}(x, y, t)=\left\{\begin{array}{lc}
1 & \text { if } M H H_{\tau}\left(x, y, P_{i}\right) \geq 1 \\
0 & \text { otherwise }
\end{array}\right.
$$

These MHH and MEH images are considered for the recognizing various activities. However, instead of these eight images, we could consider another representation, called Motion Geometric Distribution (MGD) computed by,

$$
M G D=\left\{\sum_{x} M E H\left(x, y, P_{i}\right), \sum_{y} M E H\left(x, y, P_{i}\right)\right\}
$$

\section{RECOGNITION METhodology}

In this comparative experiment, we consider some aerobics which are complex in nature [28]. Fig. 6 shows six activities that are taken from a frontal-view camera without any calibration process or any special markers from six different subjects. The actors performed the exercises indoor with almost the same illumination level, without any special arrangements and therefore, the frame numbers are varied for the same activity by various persons. Also the dress, height, etc. of the persons were different. The frame has resolution of $320 \times 240$ pixels.

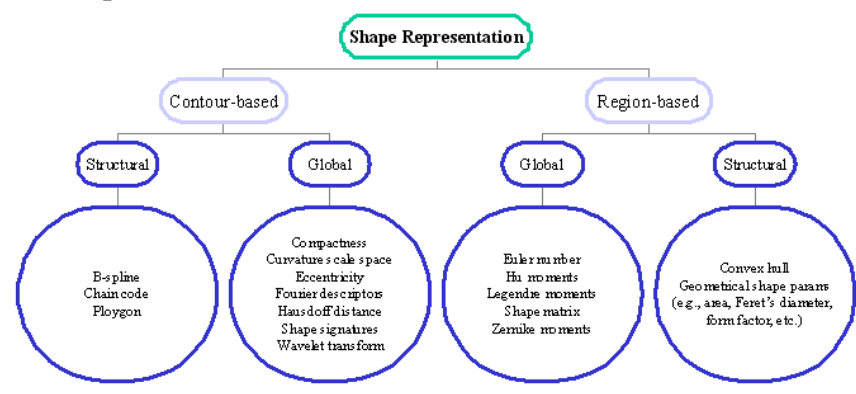

Figure 7. Various approaches for shape representation. Region-based global $\mathrm{Hu}$ moments are considered in this paper.

From the final template images, the corresponding feature vectors are computed by employing the seven $\mathrm{Hu}$ invariants [29], which are widely used for shape representation. Fig. 7 shows various other options for shape representation based on 
[7,30]. However, we have considered $\mathrm{Hu}$ geometric moments as it is fast and accurate to represent shapes than most of the other methods. Moreover, the basic MHI method along with other recent papers have employed these $\mathrm{Hu}$ invariants for calculating feature vectors. We have also tried with Zernike moments but failed to achieve good recognition results. For the MHI method, for each action, seven higher order $\mathrm{Hu}$ invariants are computed for history and energy images and therefore, a 14-D feature vector is created for each activity. In the same way, for the MMHI representation, we compute a 14$\mathrm{D}$ feature vector. In this experiment, we implemented the HMHH representation with four patterns (i.e., $M=4$ ) because patterns more than the first four have almost no information to employ further for calculating feature vectors. Therefore, for these four patterns, using the HMHH representation, we compute a 28-D feature vector (for each pattern, we compute seven higher order moments). In another experiment, we also computed the binarized energy images of the four patterns of the history images. But we have not achieved any difference to consider the energy versions for calculating feature vectors. However, for the four directional history and energy components, we need to compute seven $\mathrm{Hu}$ moments for each of the eight components. Moreover, to cover the overwriting issue significantly, we also computed the normalized $0^{\text {th }}$ order moments. E.g., for $H_{\tau}^{+x}$ for an image $f(x, y)$, the $0^{\text {th }}$ order moment is:

$$
m_{00}\left(H_{\tau}^{+x}\right)=\sum \sum f(x, y)
$$

Hence, the normalized $0^{\text {th }}$ order moment for $H_{\tau}^{+x}$ can be achieved by employing the following equation:

$$
\overline{m_{00}\left(H_{\tau}^{+x}\right)}=\frac{m_{00}\left(H_{\tau}^{+x}\right)}{m_{00}\left(H_{\tau}^{+x}\right)+m_{00}\left(H_{\tau}^{-x}\right)+m_{00}\left(H_{\tau}^{+y}\right)+m_{00}\left(H_{\tau}^{-y}\right)}
$$
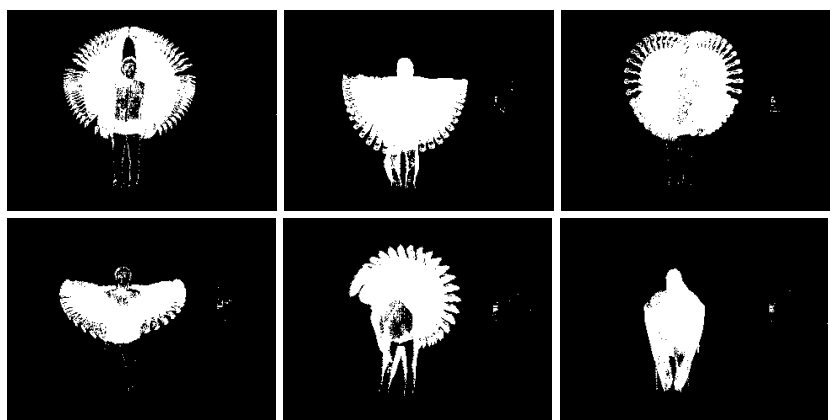

(a) The MEI images for six activities

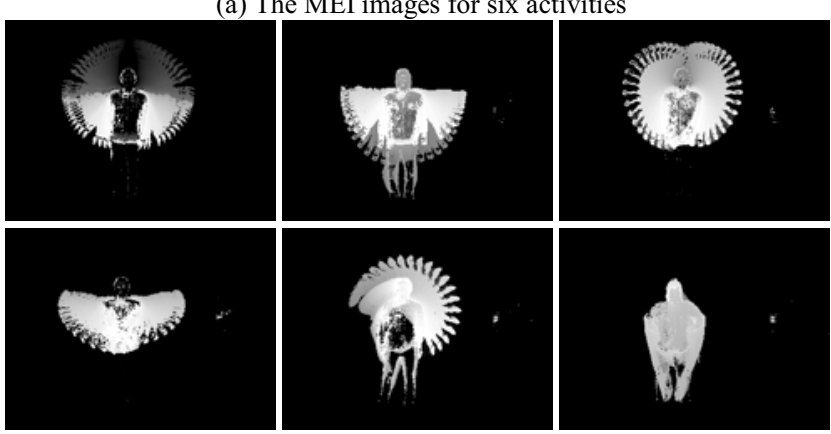

(b) The MHI images for six activities

Figure 8. Motion history images and energy images for six activities.
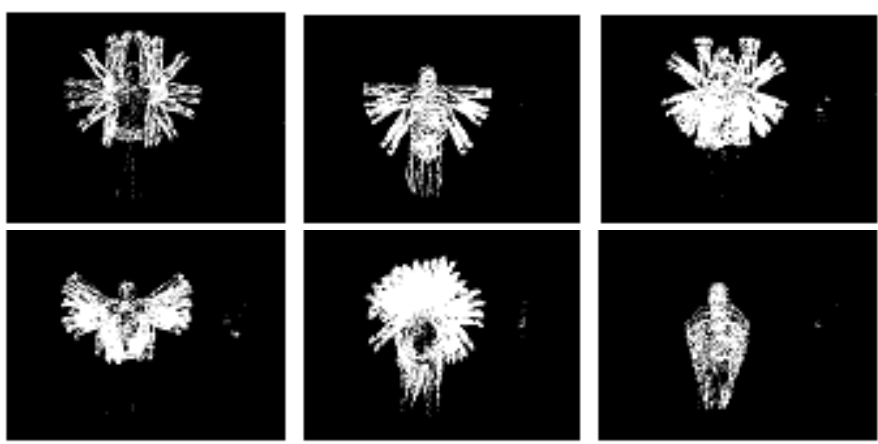

Figure 9. Energy images of six activities using the MMHI representation.
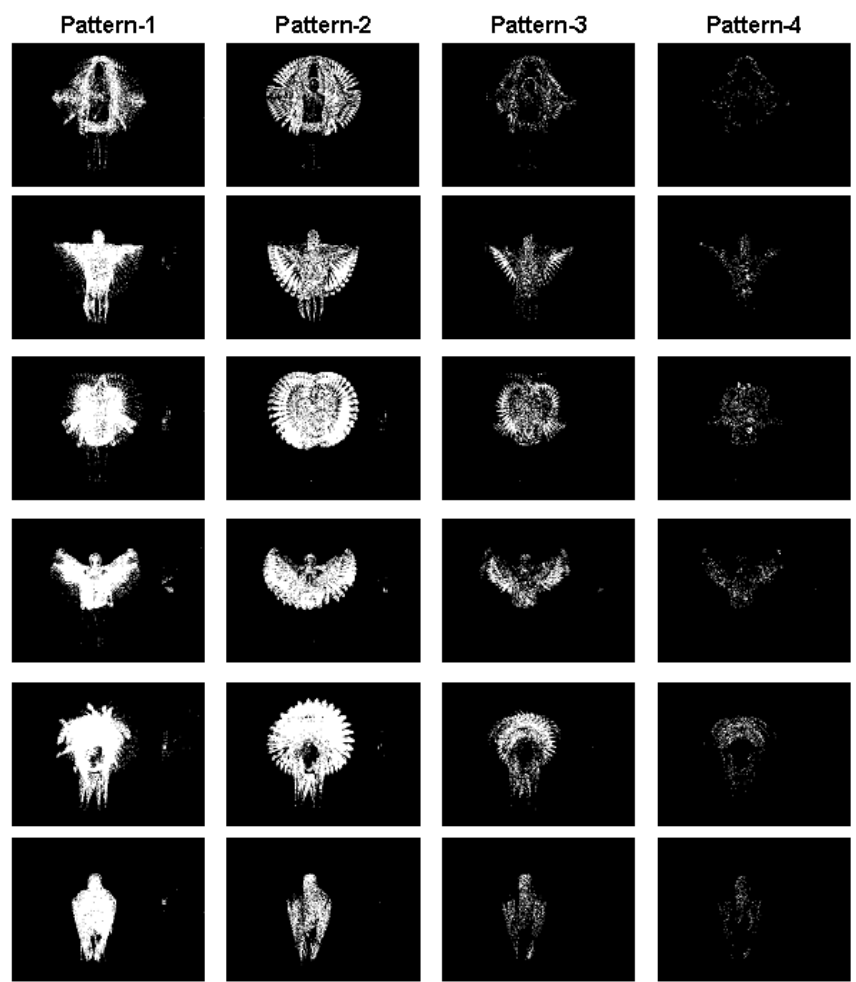

Figure 10. Four patterns for activities (images in each row represent activity 1 through 6) employing HMHH representation.

The $0^{\text {th }}$ order moments provide the total object area or mass, and hence provide important cues for motion region. Therefore, for the DMHI, we estimate eight normalized $0^{\text {th }}$ order moments for the eight templates (DMHIs and DMEIs) to get a 64-dimensional feature vector (i.e., $7 \mathrm{Hu}$ moments for each template in addition to normalized $0^{\text {th }}$ order moments). For classification, we use the $k$-nearest neighbor $(k \mathrm{NN})$ classifier [31,24]. The employed $k \mathrm{NN}$ algorithm is very straightforward. For a 'test' sample, it uses a distance metric to compute which $k$ (labeled) training samples are nearest to the sample in question and then casts a majority vote on the labels of the nearest neighbors to decide the class of the test sample.

Parameters of interest are the distance metric being used and $k$, the number of neighbors to consider. The distance measure for two feature sets $\left(\Gamma\right.$ and $\Gamma^{o}$ are the feature sets for a training sample and the test sample respectively) that performed the best is the simple Euclidian distance measure: 


$$
D\left(\Gamma, \Gamma^{\circ}\right)=\sqrt{\sum_{i=1}^{d}\left(\Gamma_{i}^{\circ}-\Gamma_{i}\right)^{2}}
$$

where, $d$ is the dimensionality of the feature space. In this paper, both training and testing samples have equal number of features. For partitioning scheme, we employ leave-one-out cross-validation scheme. This means that out of $N$ samples from each of the $c$ classes per database, $N-1$ of them are used to train (design) the classifier and the remaining one to test it [31]. This process is repeated $N$ times, each time leaving a different sample out. Therefore, all of the samples are ultimately used for testing. This process is repeated and the resultant recognition rate is averaged.

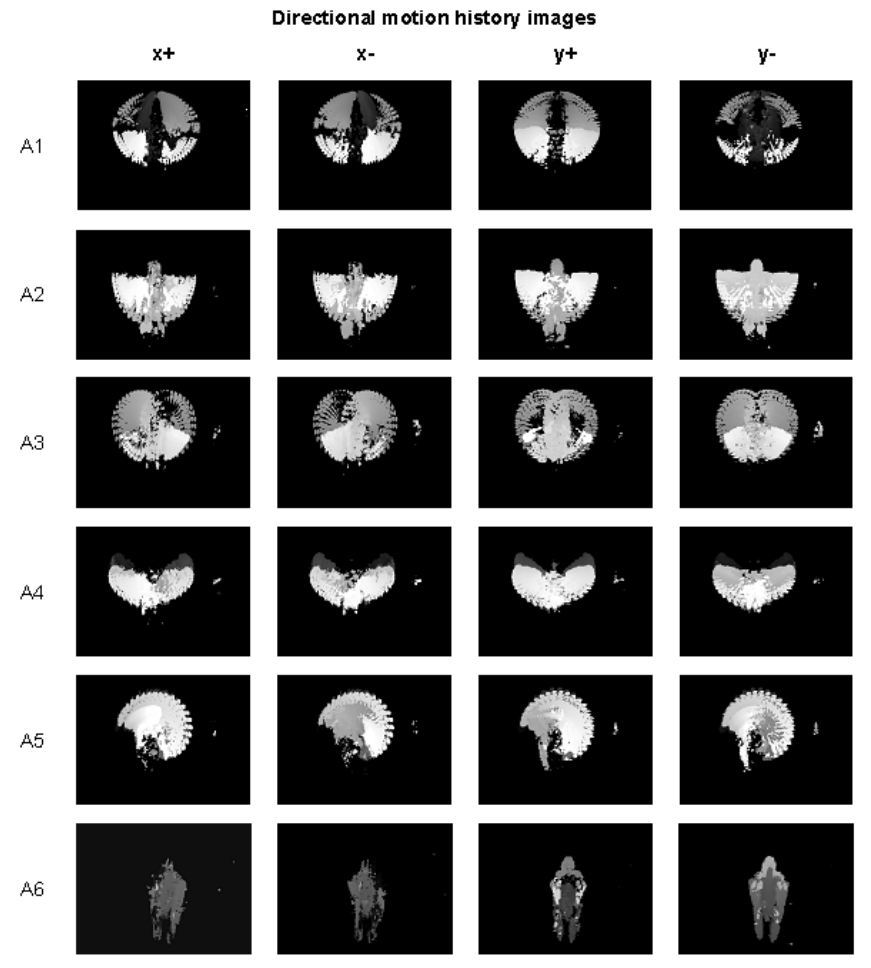

Figure 11. Directional motion history images for six activities.

\section{RESUlTS AND ANALYSIS}

\section{A. Comparative Analysis of Recognition Results}

In this paper, we presented the MHI, the MMHI, the $\mathrm{HMHH}$ and the DMHI representations for recognizing aerobics dataset. Fig. 8 shows the MEIs and the MHIs for six activities of Fig. 6. Fig. 9 illustrates the final energy images employing MMHI method for the six activities. Fig. 10 depicts the four patterns for HMHH representation for six activities. We can note that pattern-4 of Fig. 10 has almost no information and hence we did not consider pattern-5 or more for this experiment (even we could ignore pattern- 4 if motion information is not enough). Fig. 11 presents the directional history images for four different directions - namely, up, down, left and right for each activity.

Table I shows the comparative recognition results for the four methods for $k$ 's value as 1 and 3 in the nearest-neighbor classifier. For every activity (in the Tables, these six activities are represented as $\mathrm{A} 1$ to $\mathrm{A} 6$ ), the recognition results with our
DMHI representation are very satisfactory. With $k$ 's value as 1, for the DMHI method, we have achieved more than $94 \%$ recognition result. This is very good result considering the complexity of the activities. Though the HMHH representation achieved better results than the MHI and the MMHI representations, the performance of the former is unacceptable too as it has also achieved poor recognition rate.

TABLE I. COMPARATIVE RECOGNITION RATES FOR VARIOUS AEROBIC DATASETS. ALL RESULTS ARE PRESENTED IN \%.

\begin{tabular}{|c|c|c|c|c|c|c|c|c|}
\hline \multirow{2}{*}{} & \multicolumn{2}{|c|}{ MHI } & \multicolumn{2}{c|}{ MMHI } & \multicolumn{2}{c|}{ HMHH } & \multicolumn{2}{c|}{ DMHI } \\
\cline { 2 - 9 } & $k=1$ & $k=3$ & $k=1$ & $k=3$ & $k=1$ & $k=3$ & $k=1$ & $k=3$ \\
\hline A1 & 50.0 & 50.0 & 83.4 & 83.4 & 66.7 & 66.7 & 100 & 100 \\
\hline A2 & 16.7 & 16.7 & 66.7 & 33.4 & 83.4 & 83.4 & 100 & 83.4 \\
\hline A3 & 66.7 & 66.7 & 33.4 & 16.7 & 50.0 & 50.0 & 83.4 & 83.4 \\
\hline A4 & 33.4 & 16.7 & 16.7 & 33.4 & 33.4 & 33.4 & 100 & 100 \\
\hline A5 & 83.4 & 83.4 & 33.4 & 83.4 & 100 & 100 & 83.4 & 83.4 \\
\hline A6 & 83.4 & 83.4 & 50.0 & 50.0 & 66.7 & 66.7 & 100 & 100 \\
\hline RR & 55.6 & 52.8 & 47.3 & 50.0 & 66.7 & 66.7 & 94.4 & 91.7 \\
\hline
\end{tabular}

TABLE II. CM FOR THE MHI FOR AEROBICS DATA; TRACES 333.6.

\begin{tabular}{|c|c|c|c|c|c|c|}
\hline & A1 & A2 & A3 & A4 & A5 & A6 \\
\hline A1 & $\mathbf{5 0 . 0}$ & 16.7 & 0 & 16.7 & 16.7 & 0 \\
\hline A2 & 50.0 & $\mathbf{1 6 . 7}$ & 0 & 16.7 & 16.7 & 0 \\
\hline A3 & 0 & 0 & $\mathbf{6 6 . 7}$ & 0 & 33.4 & 0 \\
\hline A4 & 33.4 & 16.7 & 0 & $\mathbf{3 3 . 4}$ & 0 & 16.7 \\
\hline A5 & 16.7 & 0 & 0 & 0 & $\mathbf{8 3 . 4}$ & 0 \\
\hline A6 & 0 & 0 & 0 & 16.7 & 0 & $\mathbf{8 3 . 4}$ \\
\hline
\end{tabular}

TABLE III. CM FOR THE MMHI FOR AEROBICS DATA; TRACES 283.6.

\begin{tabular}{|c|c|c|c|c|c|c|}
\hline & A1 & A2 & A3 & A4 & A5 & A6 \\
\hline A1 & $\mathbf{8 3 . 4}$ & 16.7 & 0 & 0 & 0 & 0 \\
\hline A2 & 0 & $\mathbf{6 6 . 7}$ & 0 & 16.7 & 0 & 16.7 \\
\hline A3 & 0 & 0 & $\mathbf{3 3 . 4}$ & 16.7 & 50.0 & 0 \\
\hline A4 & 0 & 33.4 & 33.4 & $\mathbf{1 6 . 7}$ & 0 & 16.7 \\
\hline A5 & 0 & 0 & 66.7 & 0 & $\mathbf{3 3 . 4}$ & 0 \\
\hline A6 & 16.7 & 16.7 & 0 & 16.7 & 0 & $\mathbf{5 0 . 0}$ \\
\hline
\end{tabular}

TABLE IV. CM FOR THE HMHH FOR AEROBICS DATA; TRACES 416.9.

\begin{tabular}{|c|c|c|c|c|c|c|}
\hline & A1 & A2 & A3 & A4 & A5 & A6 \\
\hline A1 & $\mathbf{5 0}$ & 0 & 0 & 16.7 & 0 & 33.4 \\
\hline A2 & 16.7 & $\mathbf{8 3 . 4}$ & 0 & 0 & 0 & 0 \\
\hline A3 & 0 & 0 & $\mathbf{6 6 . 7}$ & 16.7 & 0 & 16.7 \\
\hline A4 & 16.7 & 16.7 & 16.7 & $\mathbf{3 3 . 4}$ & 16.7 & 0 \\
\hline A5 & 0 & 0 & 0 & 0 & $\mathbf{1 0 0}$ & 0 \\
\hline A6 & 16.7 & 0 & 0 & 0 & 0 & $\mathbf{8 3 . 4}$ \\
\hline
\end{tabular}

TABLE V. CM FOR THE DMHI METHOD; TRACES 566.8 .

\begin{tabular}{|c|c|c|c|c|c|c|}
\hline & A1 & A2 & A3 & A4 & A5 & A6 \\
\hline A1 & $\mathbf{1 0 0}$ & 0 & 0 & 0 & 0 & 0 \\
\hline A2 & 0 & $\mathbf{1 0 0}$ & 0 & 0 & 0 & 0 \\
\hline A3 & 0 & 0 & $\mathbf{8 3 . 4}$ & 0 & 16.7 & 0 \\
\hline A4 & 0 & 0 & 0 & $\mathbf{1 0 0}$ & 0 & 0 \\
\hline A5 & 0 & 0 & 16.7 & 0 & $\mathbf{8 3 . 4}$ & 0 \\
\hline A6 & 0 & 0 & 0 & 0 & 0 & $\mathbf{1 0 0}$ \\
\hline
\end{tabular}

Tables II $\sim$ V show the confusion matrices $(\mathrm{CM})$ for the four methods for $k$ 's value as 1 . The confusion matrix shows the motion label (vertical) versus the classification results (horizontal). Each cell $(p, q)$ in the tables shows the percentage 
of class $p$ action being recognized as class $q$. The trace of the confusion matrix shows the percentage sum of the correctly recognized action while the remaining cells show the percentage of misclassification.

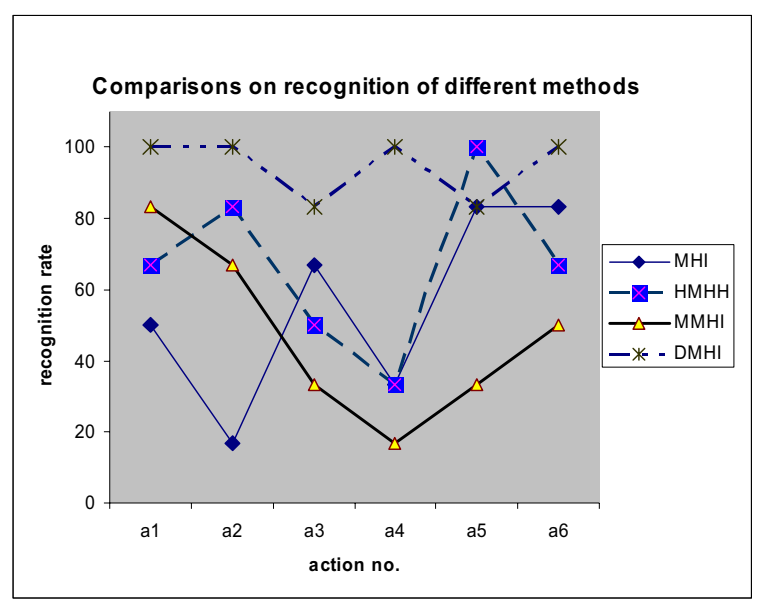

Figure 12. Graphical representation for the recognition results.

Fig. 12 depicts these results in a graph that demonstrates that the DMHI method visibly outshines other methods to solve the motion overwriting problem due to self-occlusion.

\section{B. Robustness Analysis of the DMHI Method}

To show its robustness, more experiments were accomplished with the DMHI method. We compared the results with the MHI method only in this case. Ten different exercises are considered for this comparison and as Table VI shows, the DMHI shows satisfactory recognition results (e.g., $93.8 \%$ with $k=1$ ) even with more activities. Note that the numbers of frames are not taken equal for each activity for each person. These activities have different number of frames (as shown in Fig. 13).

TABLE VI. AVERAGE RECOGNITION RESULTS FOR MHI AND DMHI.

\begin{tabular}{|c|c|c|c|}
\hline MHI (k=1) & MHI (k=3) & DMHI $(\mathbf{k}=\mathbf{1})$ & DMHI $(\mathbf{k}=\mathbf{3})$ \\
\hline 46.3 & 46.3 & 93.8 & 92.5 \\
\hline
\end{tabular}

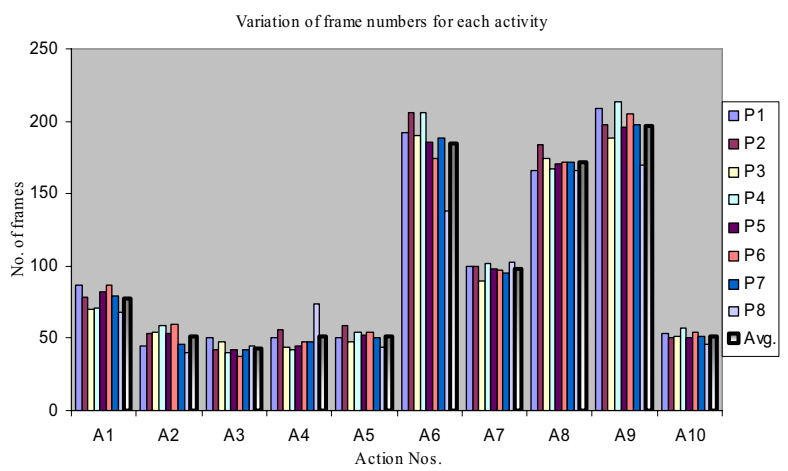

Figure 13. Variation of frame numbers for the 10 activities by 8 different subjects. The average frame numbers for each activity are also shown as Avg.

To demonstrate the performance of the DMHI method, we also did experiment with low-resolution datasets, because motion recognition from low-resolution video sequences is very difficult task and sometimes deemed to be impossible. In low-resolution image, much significant motion information and their correlations are missing from the image sequences [33]. Fig. 14 shows the concept of the missing information.
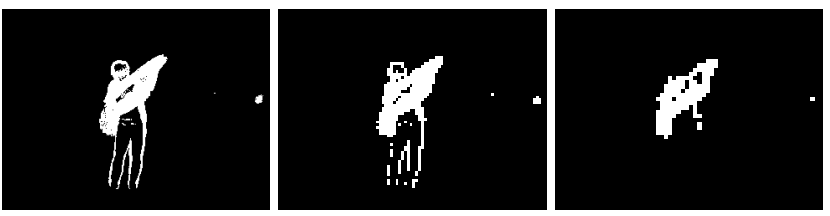

Figure 14. Typical energy images for an action for low-resolution images: $320 \times 240,96 \times 72$ and $80 \times 60$ pixel resolutions.

In our experiment with low-resolution image sequences of the above-mentioned Japanese radio exercises, we achieve more than $90 \%$ recognition results for resolution of $320 \times 240$, $160 \times 120$ until $96 \times 72$ pixels. This is indeed a significant performance of the DMHI method, because up to this very low-resolution level, other methods can not show any good performance [33]. While studying the recognition of very lowresolution video sequences, we find that for the MHI and the DMHI methods, up to $96 \times 72$ pixel resolution level, we could achieve satisfactory recognition results, while below this resolution, due to the loss in image information, we get poor result. This shows that the DMHI method can be applicable even to very low-resolution video recognition.
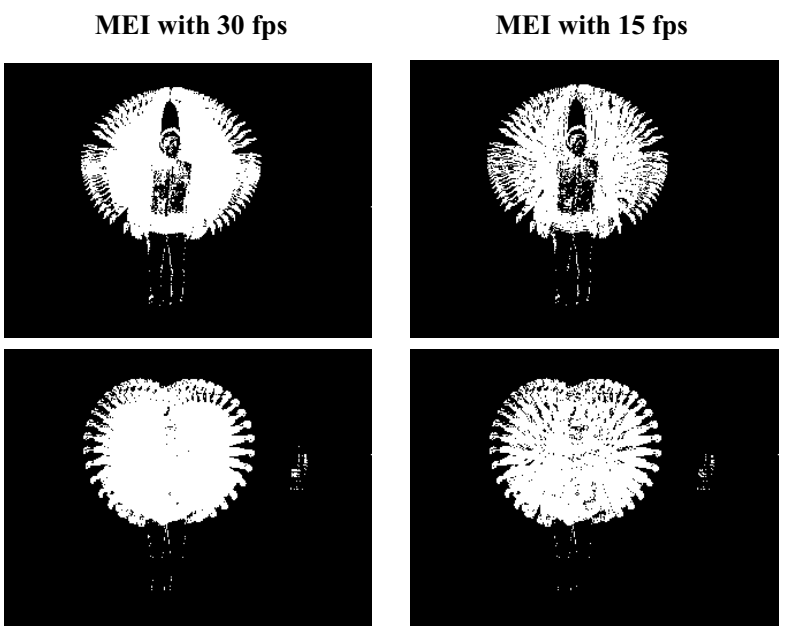

Figure 15. The MEI components for two actions with $30 \mathrm{fps}$ and faster $15 \mathrm{fps}$.

Moreover, in order to demonstrate the robustness of the DMHI against different speeds of actions, we considered various combinations of ten aerobics from eight subjects. Table VII shows the recognition results for various combinations of datasets [35]. With the 'PA_66 dataset' and $15 \mathrm{fps}$, we achieve lower recognition result for Activity-6. Here, ' $P$ ' denotes 'Person' and 'A' denotes 'Activity'; hence this PA_66 dataset consists of six activities from six persons. Similarly, PA_810 means this dataset comprises with 10 activities from 8 different individuals. Initially, we considered two different speeds for the video sequences - 30 frames per second (fps) and 15 fps. Hence, by considering 15 fps, i.e., taking half of the frames compared to $30 \mathrm{fps}$ for a period, we 
have lost some important correlations among the pixels. This is a reason to have a bit lower recognition rates with $15 \mathrm{fps}$. Fig. 15 shows some templates of energy images for $30 \mathrm{fps}$ and $15 \mathrm{fps}$. For $15 \mathrm{fps}$, some missing information is visible (as black spots in the right column of Fig. 15).

TABLE VII. RECOGNITION RATES (IN \%) OF AEROBIC DATASETS WITH VARIOUS SPEEDS AND COMBINATIONS. NOTE THAT THE LAST ROW IS ONLY WITH THE MHI; OTHERS ARE WITH THE DMHI METHOD.

\begin{tabular}{|c|c|c|}
\hline Dataset & Testing Condition & Rec. Rate \\
\hline \multirow{2}{*}{ PA_810 } & $30 \mathrm{fps}$ & 93.8 \\
\hline & $15 \mathrm{fps}$ & 78.8 \\
\hline \multirow{2}{*}{$P A \_66$} & $30 \mathrm{fps}$ & 94.4 \\
\hline & $15 \mathrm{fps}$ & 86.2 \\
\hline \multirow[b]{2}{*}{$P A \_86$} & $30 \mathrm{fps}$ & 95.9 \\
\hline & $\begin{array}{c}\text { Training } 30 \mathrm{fps} \\
\text { Testing: } 20 \% \text { less data for a } 1 \sim \mathrm{a} 4\end{array}$ & 83.4 \\
\hline \multirow{2}{*}{$\begin{array}{c}P A \_810 \\
\text { (mixed data) }\end{array}$} & $\begin{array}{c}\text { Training } 30 \mathrm{fps} \\
\text { Testing: } 20 \% \text { less data for } \mathrm{p} 1 \sim \mathrm{p} 4\end{array}$ & 82.5 \\
\hline & $\begin{array}{l}\text { Training and Testing: } \\
20 \% \text { less data for } \mathrm{p} 1 \sim \mathrm{p} 4\end{array}$ & 82.5 \\
\hline \multirow{2}{*}{ With MHI } & $30 \mathrm{fps}$ & 55.6 \\
\hline & $15 \mathrm{fps}$ & 38.9 \\
\hline
\end{tabular}

For more analysis, we also implement the basic MHI method with $15 \mathrm{fps}$, and the recognition results are dropped than earlier. Note that these aerobics are not slow and the exercise sequences are taken by various subjects without considering any specific pace of the actions. Fig. 16 graphically depicts the performances in different framesets and the DMHI performs very good results than the MHI.

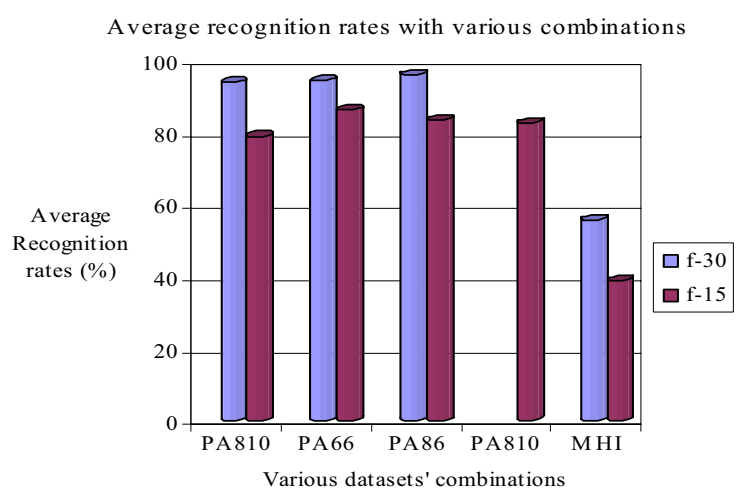

Figure 16. Graphical representation explicitly demonstrates that the recognition rates are agreeable even for $15 \mathrm{fps}$ for the DMHI. However, the recognition rates for the $\mathrm{MHI}$ method are lower.

Apart from the above issues, we also investigated on the reduction of feature vectors for shape representation. In another experiment, we consider different reduced feature vector sets in order to see the performance and robustness of our method. Instead of using higher seven order invariants and the normalized $0^{\text {th }}$ order moments, we tried with the following different sets (every sub-equation in Eq. 21 denotes feature vectors of Fig. 17 as 56-D, 40-D, 36-D, 32-D and 24-D respectively). For example, $F V_{56 D}$ consists of seven invariants for both history and energy templates, whereas, $F V_{32 D}$ set is composed with seven invariants for history images along with the normalized $0^{\text {th }}$ order moments of the energy images.
Therefore, these feature vectors can significantly reduce the computational cost without sacrificing the recognition rates.

$$
\begin{aligned}
& F V_{56 D}=(\forall h)\left(I_{1 \rightarrow 7}\right) \cup(\forall e)\left(I_{1 \rightarrow 7}\right) . \\
& F V_{40 D}=(\forall h)\left(I_{1 \rightarrow 5}\right) \cup(\forall e)\left(I_{1 \rightarrow 5}\right) . \\
& F V_{36 D}=(\forall h)\left(I_{1 \rightarrow 7}\right) \cup(\forall h)\left(\overline{m_{00}^{\sigma}}\right) \cup(\forall e)\left(\overline{m_{00}^{\sigma}}\right) . \\
& F V_{32 D}=(\forall h)\left(I_{1 \rightarrow 7}\right) \cup(\forall e)\left(\overline{m_{00}^{\sigma}}\right) . \\
& F V_{24 D}=(\forall h)\left(I_{1 \rightarrow 3}\right) \cup(\forall e)\left(I_{1 \rightarrow 3}\right) .
\end{aligned}
$$

Average recognition rates with various combinations

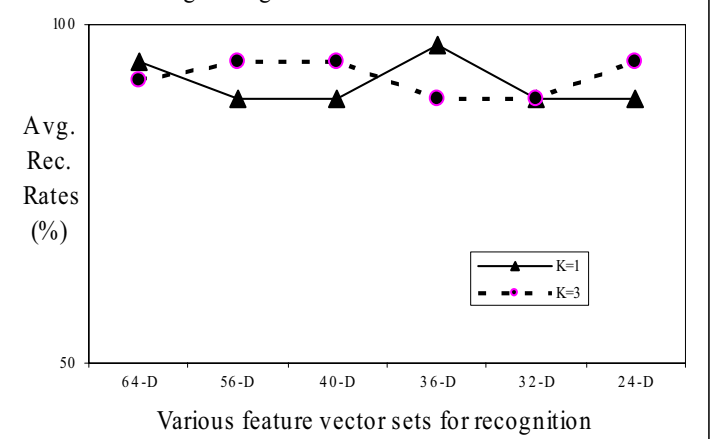

Figure 17. Comparative recognition results with reduced feature vector sets while considering the DMHI method.

From Fig. 17, we can vividly notice that the average recognition rates are still in satisfactory ranges even with lower-dimensional feature vectors. We find it a wonderful development with the DMHI method, because in all other approaches that have employed $\mathrm{Hu}$ invariants in their works, have calculated the seven invariants, even though higher invariants are more noise-prone. In our case, the deployment of lower dimensional feature vectors also can produce good recognition results. Moreover, our experimental results and analysis find that both history and energy images are required for recognition by using the DMHI method. Apart from these, we also accomplished several experiments with the HMHH method and the MHI method with various different feature vector combinations (instead of using the seven invariants). From these results, we find that it is not required to employ the higher order invariants as well as the usage of the first two or three invariants demonstrate good recognition results. It is known that the first two $\mathrm{Hu}$ invariants provide scale and translation invariance, whereas next four $\mathrm{Hu}$ invariants ensure rotation with reflection invariance. And the last seven invariant provides reflection discrimination in its sign. Therefore, unless the rotation or reflection invariance is required, it is preferable not to employ the higher invariants. Moreover, it is evident from the calculation that higher invariants are computationally costlier, and at the same time, these are more prone to noise. Hence, the concept of reduced feature vector will promote for faster recognition strategy with the DMHI approach and others too.

\section{CONCLUSION}

This paper presents various template-based solutions to the motion self-occlusion problem in action analysis and recognition. The basic MHI method is very simple to calculate 
and hence many researchers employed this method or its variants for various action/gesture recognition, motion analysis, etc., with different datasets. The motion selfocclusion problem is addressed and solved with satisfactory recognition rate with our DMHI method. We compared the DMHI representation with the MHI, the Multi-level Motion History Image (MMHI) method and the Hierarchical Motion History Histogram (HMHH) approaches. The latter two approaches claim to be better in solving overwriting problem. However, these representations failed to perform well compared to our method. This paper also presented the performances of the DMHI in low-resolution video sequences. We also presented reduced feature vector sets.

Though the performance of the DMHI representations and the employed feature vectors for recognition are satisfactory, we find that this method can not perform well or faces difficulties in some cases. When two or more than two persons are in-view, this method (along with other presented methods in this paper) can not recognize properly, especially when all of them are moving in different directions. Also, like the MHI, it can not recognize properly if the person is walking towards the camera's optical axis or if it moves something like diagonal directions. For these types of actions, we need to consider a bit different feature vector sets. For walking towards or off the camera, if we can exclude the energy images, the history image can separate and recognize the actions properly. These problems will remain for future research so that the method can be made a robust one for different types of motions or activities. Another important pair of activities are running and walking across the optical axis and to recognize these is very crucial for video surveillance. These are difficult to recognize in a simple manner. Currently, we are working by considering time measurement to differentiate both actions, so that these could be recognized separately. Note that using the DMHI, it is far easier to understand the motion flow, i.e., the direction of the motion running or walking easily. This information is very useful for behavior understanding and analyzing.

\section{ACKNOWLEDGEMENT}

Authors are grateful to the students who took part in the exercises. The preliminary version of the paper was presented at the $11^{\text {th }}$ International Conference on Computer and Information Technology [34].

\section{REFERENCES}

[1] A. Bobick and J. Davis, "The recognition of human movement using temporal templates", IEEE Trans. on PAMI, vol. 23, no. 3, pp. 257-267, March 2001.

[2] Md. Atiqur Rahman Ahad, J.K. Tan, H.S. Kim, and S. Ishikawa, "Human activity recognition: various paradigms", Int. Conf. on Control, Automation and Systems, pp. 1896-1901, Oct. 2008.

[3] D. Gavrilla, "The visual analysis of human movement: a survey", Computer Vision and Image Understanding, vol. 73, pp. 82-98, 1999.

[4] M. Pantic, A. Pentland, A. Nijholt, and T.S. Hunag, "Human computing and machine understanding of human behavior: a survey", Int. Conf. on Multimodal Interfaces, pp. 239-248, 2006.
[5] R. Poppe, "Vision-based human motion analysis: an overview", Computer Vision and Image Understanding, vol. 108, no. 1-2, pp. 4-18, Oct. 2007.

[6] Md. Atiqur Rahman Ahad, T. Ogata, J.K. Tan, H.S. Kim, and S. Ishikawa, "Motion recognition approach to solve overwriting in complex actions", $8^{\text {th }}$ Int. Conference on Automatic Face and Gesture Recognition, Amsterdam, 6 pages, Sept. 2008.

[7] V. Kindratenko, "Development and Application of Image Analysis Techniques for Identification and Classification of Microscopic Particles", PhD Thesis, University of Antwerp, Belgium, 1997. http://www.ncsa.uiuc.edu/ kindr/phd/index.pdf

[8] H. Meng, N. Pears, and C. Bailey, "A Human Action Recognition System for Embedded Computer Vision Application", $3^{\text {nd }}$ Workshop on Embedded Computer Vision (with CVPR), pp. 1-6, June 2007.

[9] J. Davis and A. Bobick, "Virtual PAT: a virtual personal aerobics trainer", Perceptual User Interfaces, pp. 13-18, 1998.

[10] J. Davis and G. Bradski, "Real-time motion template gradients using Intel CVLib", IEEE ICCV Workshop on Frame-rate Vision, pp. 1-20, Sept. 1999.

[11] W.C. Yau, D.K. Kumar, S.P. Arjunan, and S. Kumar, "Visual speech recognition using image moments and multiresolution wavelet", Int. Conf. on Computer Graphics, Imaging and Visualization, pp. 194-199, 2006.

[12] Z. Yin and R. Collins, "Moving object localization in thermal imagery by forward-backward MHI", Third IEEE Workshop on Object Tracking and Classification in and Beyond the Visible Spectrum (OTCBVS'06), pp. 133-140, NY, June 2006.

[13] L. Li, Q. Zeng, Y. Jiang, and H. Xia, "Spatio-temporal motion segmentation and tracking under realistic condition", Int'l Symposium on Systems and Control in Aerospace and Astronautics, pp. 229-232, 2006.

[14] D. Son, T. Dinh, V. Nam, T. Hanh, and H. Lam, "Detection and localization of road area in traffic video sequences using motion information and fuzzy-shadowed sets", IEEE Int'l Symp. on Multimedia, pp. 725-732, Dec. 2005.

[15] J. Liu and N. Zhang, "Gait history image: a novel temporal template for gait recognition", IEEE Int. Conf. on Multimedia and Expo, pp. 663-666, 2007.

[16] J. Han and B. Bhanu, "Individual recognition using gait energy image", IEEE Trans. Pattern Analysis and Machine Intelligence, vol. 28, no. 2, pp. 316-322, 2006.

[17] T. Jan, "Neural network based threat assessment for automated visual surveillance", IEEE Int. Joint Conf. on Neural Networks, vol. 2, pp. 1309-1312, July 2004.

[18] K. Leman, G. Ankit, and T. Tan, "PDA-based human motion recognition system", Int. J. Software Engineering and Knowledge, vol. 2, issue 15, pp. 199-205, Apr. 2005.

[19] I. Petrás, C. Beleznai, Y. Dedeoğlu, M. Pardàs, et al., "Flexible test-bed for unusual behavior detection", ACM Int. Conf. on Image and video retrieval, pp. 105-108, 2007.

[20] V. Kellokumpu, G. Zhao, and M. Pietikäinen, "Texture based description of movements for activity analysis" Third Int. Conf. on Computer Vision Theory and Applications (VISAPP 2008), Madeira, Portugal, vol. 1, pp. 206-213.

[21] H. Meng, N. Pears, and C. Bailey, "Motion Information Combination for Fast Human Action Recognition", $2^{\text {nd }}$ International Conference on Computer Vision Theory and Applications, Spain, Mar. 2007.

[22] H. Meng, N. Pears, and C. Bailey, "Recognizing Human Actions Based on Motion Information and SVM", $2^{\text {nd }}$ IEE International Conference on Intelligent Environments, pp. 239-245, 2006. 
[23] M. Valstar, I. Patras, and M. Pantic, "Facial action recognition using temporal templates", IEEE Int. Workshop on Robot and Human Interactive Communication, pp. 253-258, Sep. 2004.

[24] M. Valstar, M. Pantic, and I. Patras, "Motion history for facial action detection in video", IEEE Int'l Conf. on Systems, Man and Cybernetics, pp. 635-640, 2004.

[25] G. Bradski and J. Davis, "Motion segmentation and pose recognition with motion history gradients", Int. J. of Machine Vision and Applications, vol. 13, no. 3, pp. 174-184, 2002.

[26] T. Ogata, J.K. Tan, and S. Ishikawa, "High-speed human motion recognition based on a motion history image and an Eigenspace", IEICE Trans. Information \& Systems, vol. E89-D, no. 1, pp. 281-289, 2006.

[27] A.A. Efros, A.C. Berg, G. Mori, J. Malik, "Recognizing Action at a Distance", ICCV, pp. 726-733, 2003.

[28] http:/gaijin-in-japan.com/2007/08/11/rajio-taiso-radio-exercise/

[29] M.K. Hu, "Visual pattern recognition by moment invariants", IRE Trans. on Info. Theory, pp. 179-187, 1962.

[30] D. Zhang and G. Lu, "Review of shape representation and description techniques", Pattern Recognition, vol. 37, pp. 1-19, 2004.

[31] A. Khotanzad and Y. H. Hong, "Invariant Image Recognition by Zernike Moments", IEEE Transaction Pattern Analysis and Machine Intelligence, vol. 12, no. 5, pp. 489-497, May 1990.

[32] W. J. Christmas, "Spatial Filtering Requirements for GradientBased Optical Flow Measurement", $9^{\text {th }}$ British Machine Vision Conference, pp. 185-194, 1998.

[33] Md. Atiqur Rahman Ahad, T. Ogata, J.K. Tan, H.S. Kim, and S. Ishikawa, "Directional motion history templates for low resolution motion recognition", $34^{\text {th }}$ Annual Conference of IEEE Industrial Electronics Society (IECON), pp. 1875-1880, Orlando, Nov. 2008.

[34] Md.A.R. Ahad, J.K. Tan, H.S. Kim, and S. Ishikawa, "Solution to motion self-occlusion problem in human activity analysis", Int. Conf. Computer and Information Tech., pp. 201-206, 2008.

[35] Md.A.R. Ahad, J.K. Tan, H.S. Kim, and S. Ishikawa, "Action Recognition with Various Speeds and Timed-DMHI Feature Vectors", Int. Conf. on Computer and Info. Tech., pp. 213-218, Dec. 2008

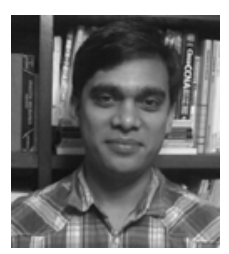

\section{Md. Atiqur Rahman AHAD}

Md. Atiqur Rahman Ahad was born is Bangladesh and has obtained B.Sc. (Hons') and Masters degrees from the Department of Applied Physics, Electronics and Communication Engineering, University of Dhaka, Bangladesh. He later received Masters degree in Computer Science (2 years) from School of Computer Science and Engineering, University of New South Wales, Australia.

He joined the University of Dhaka, Bangladesh in 2001 and continued his career as a Faculty member in various universities, and currently under study-leave from the University of Dhaka. He also served as a Casual Academic in University of New South Wales during 3 sessions in 2002 to 2004 . He is currently doing his Ph.D. course at Kyushu Institute of Technology, Japan.

Mr. Ahad is a student member of IEEE, IEEE IES and SICE. He has received the Best Student Paper Award in the International Workshop on Combinatorial Image Analysis (IWCIA), Buffalo, NY, April 2008. He has also been awarded the Biomedical Fuzzy Systems Association's Best Paper Award in the 2008 for the paper published in IJBSCHS, Vol. 13, No. 1. His present research includes human motion recognition and analysis, motion segmentation, motion tracking, etc.

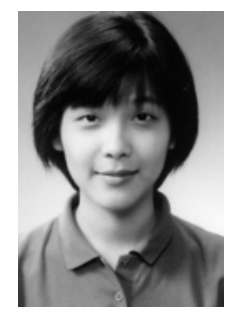

\section{Joo Kooi TAN}

Joo Kooi TAN received B.E. and M.E. degrees in Computer Science and Ph.D degree in Control Engineering from Kyushu Institute of Technology. She is presently an Assistant Professor with Department of Mechanical and Control Engineering in the same university. Her current research interests include three-dimensional shape/motion recovery, human motion analysis, human activities recognition, and applications of computer vision.

She received the SICE Kyushu Branch Young Author's Award in 1999, the AROB10th Young Author's Award in 2004, and Young Author's Award from IPSJ of Kyushu Branch in 2004. She is a member of IEEE, The Society of Instrument and Control Engineers, and The Information Processing Society of Japan.

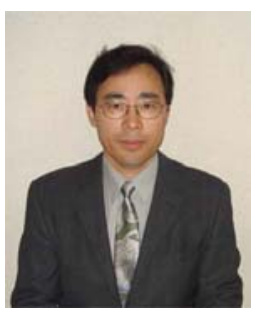

\section{Hyoungseop KIM}

Hyoungseop Kim received his B.A. degree in electrical engineering from Kyushu Institute of Technology in 1994, the Masters and Ph.D. degree from Kyushu Institute of Technology in 1996 and 2001, respectively. He is an associate professor in the Department of Control Engineering at Kyushu Institute of Technology.

His research interests are focused on medical application of image analysis. He is currently working on automatic segmentation of multi-organ of abdominal CT image, and temporal subtraction of thoracic MDCT image sets.

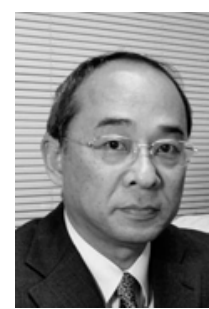

\section{Seiji ISHIKAWA}

Seiji Ishikawa obtained B.E., M.E., and D.E. from The University of Tokyo, where he majored in Mathematical Engineering and Instrumentation Physics. He joined Kyushu Institute of Technology and he is currently Professor of Department of Control \& Mechanical Engineering. Professor Ishikawa was a visiting research fellow at Sheffield University, U.K., from 1983 to 1984, and a visiting professor at Utrecht University, The Netherlands, in 1996.

His research interests include three-dimensional shape/motion recovery, human motion analysis, medical image analysis, and a multiple robots system. He is a member of IEEE, The Society of Instrument and Control Engineers, The Institute of Electronics, Information and Communication Engineers, and The Institute of Image Electronics Engineers of Japan. 